\title{
Carthami Semen Pharmacopuncture Combined with Electroacupuncture on Carpal Tunnel Syndrome: A Retrospective Case Series Study
}

\author{
Pyung-Wha Kim ${ }^{1}$, Seon Choe $^{2}$, Kyungsun Han ${ }^{1}$, Changsop Yang ${ }^{1}$, Jinbok Lee ${ }^{3}$, Sungha Kim ${ }^{1}$, \\ Minseop Shin ${ }^{4}$ * \\ ${ }^{1}$ Clinical Medicine Division, Korea Institute of Oriental Medicine, Daejeon, Republic of Korea \\ ${ }^{2}$ Division of Biomedical Informatics, Seoul National University College of Medicine, Seoul, Republic of Korea \\ ${ }^{3}$ Lee Jinbok Korean Medicine Clinic, Jeonju, Republic of Korea \\ ${ }^{4}$ Chuk-u Acupuncture \& Moxibustion Korean Medicine Clinic, Jeonju, Republic of Korea
}

Received May 31, 2021

Reviewed May 31, 2021

Accepted June 4, 2021

*Corresponding Author

Sungha Kim

Clinical Medicine Division, Korea

Institute of Oriental Medicine, Daejeon

34054, Republic of Korea

Tel: +82-42-868-9345

E-mail: bozzol@kiom.re.kr

\section{Minseop Shin}

Chuk-u Acupuncture \& Moxibustion

Korean Medicine Clinic, Jeonju 55056,

Republic of Korea

Tel: +82-63-228-7579

E-mail: kahn815@hanmail.net
While carpal tunnel syndrome (CTS) is the most common entrapment neuropathy affecting the wrist, resulting in substantial physical, psychological, and economic effects, there is no gold standard therapy for CTS. In this case series study, we aimed to report CTS patients treated with Carthami Semen Pharmacopuncture (CSP) and electroacupuncture (EA) showing improvements in their symptoms, and the combinatorial effects of CSP and EA. We collected medical records of CTS outpatients who received CSP and EA at Chuku Acupuncture \& Moxibustion Korean Medicine Clinic from August 2017 to September 2018. The outcome measures were the visual analog scale (VAS) for pain, paresthesia, the Korean version of the Boston carpal tunnel questionnaire (K-BCTQ) score, and changes in nocturnal pain, Tinel sign, and Phalen's test. We included patient satisfaction at the completion of all treatments. 17 patients were included for this case series study. After treatment, VAS for pain decreased significantly from $50.41 \pm 16.19$ to $9.59 \pm 9.46$, VAS for paresthesia also decreased significantly from $63.50 \pm 11.49$ to $14.75 \pm 12.97$, and KBCTQ symptom severity scale decreased from $2.48 \pm 0.68$ to $1.89 \pm 0.70$ (all $p<0.001$ ). Nocturnal pain, Tinel signs, and Phalen's test showed improvements after all the treatments. All the patients reported favorable overall satisfaction with the treatments, and $69.23 \%$ wanted future pharmacopuncture treatments if CTS recurred. No complications were detected. The combination of CSP and EA could be an effective and safe option in treating CTS.

Keywords: carpal tunnel syndrome, carthami semen pharmacopuncture, electroacupuncture, case-series study

\section{ABBREVIATIONS}

$\mathrm{AD}$, Anteroposterior diameter; AE, Adverse event; CSA, Cross-sectional area; CSP, Carthami Semen pharmacopuncture; CTS, Carpal tunnel syndrome; EA, Electroacupuncture; KBCTQ, Korean version of Boston carpal tunnel questionnaire; KGMP, Korea good manufacturing practice; KM, Korean medicine; TCL, Transverse carpal ligament; TD, transverse diameter; VAS, Visual analogue scale KGMP.

\section{INTRODUCTION}

Carpal tunnel syndrome (CTS) is the most common nerve entrapment syndrome at the level of the hand [1]. CTS prevalence varies widely in the general population, ranging from $0.3 \%$ to $5 \%$ due to the diagnostic criteria in different studies because the choice of broad (history or Phalen's test) or strict (sensory or motor deficits) criteria leads to variability in the prevalence findings $[1,2]$. In South Korea, one study reported $0.75 \%$ of 
CTS prevalence in the general urban population [3].

Typical CTS symptoms are mainly hand pain, characterized as the proximal pain and sensory symptoms, and numbness, tingling, and muscular dysfunction, etc. [4]. Those symptoms are caused by compression of the median nerve in a spacelimited osteofibrous canal (i.e., the carpal tunnel), and edema, tendon inflammation, and repetitive manual activities can contribute to it [1]. Furthermore, recent studies indicate that CTS patients suffer from substantial physical, psychological (e.g., anxiety and lower health-related quality of life) and economic (e.g., loss of earnings) consequences $[1,5]$.

Conventional treatments for CTS are conservative treatments such as splinting, local steroid injection, and oral corticosteroids, and surgical intervention such as decompressive surgery $[2,6]$. While local steroid injections can improve CTS conditions quickly, there are potential risks like persistent skin depigmentation or subcutaneous atrophy [6-8]. Decompressive surgery is more effective long-term than non-surgical conservative treatments [1]; however, side effects (e.g., formation of a painful neuroma, tender or hypertrophic scar, etc.) were commonly reported in the surgical group [9].

Considering the risk of complications, acupuncture combined with herbal extract and electroacupuncture (EA) can be effective and safe interventions to treat CTS $[2,10,11]$. Phamacopuncture is a clinical technique that involves injecting a small amount of refined herbal extract into acupoints. Among Korean Medicine (KM) therapies, Carthami Semen pharmacopuncture (CSP) showed short-term symptom-relieving effects in some CTS cases $[12,13]$. Moreover, in clinical fields in Korea, pharmacopuncture is frequently used and combined with acupuncture or electroacupuncture. However, the combinatorial effects of CSP and EA have never been reported in previous studies, despite its prevalent clinical use. Hence, in this study, we aimed to report case series of CTS who received CSP combined with EA, showing improvements in CTS symptoms and the combinatorial effects of CSP and EA.

\section{CASE REPORT}

The medical records of all the outpatients, who were treated for CTS in Chuk-u Acupuncture \& Moxibustion Korean Medicine Clinic (Jeonju, South Korea) from August 2017 to September 2018, were collected. We included cases based on the following eligibility criteria: (1) patients diagnosed with CTS by KM doctors [The CTS- 6 score of $\geq 12$ points [14]; numb- ness predominantly or exclusively in median nerve distribution (3.5 points), nocturnal symptoms (4 points), Thenar atrophy or weakness (5 points), positive Phalen's test (5 points), loss of 2-point discrimination ( $>5 \mathrm{~mm}$ ) (4.5 points), positive Tinel sign (4 points). If necessary, an ultrasound observation for the cross-sectional area of the median nerve supported the diagnosis of CTS $[6,15]$. (2) age $\geq 19$ years old. We excluded patients for whom the complete data records were not available, who were pregnant or lactating women, who had cardiovascular or kidney disease, and patients taking medication due to impaired liver or kidney, mental illness, and alcoholism.

The present study was approved by the Institutional Review Board of the Korea Institute of Oriental Medicine (I-1904/003001). Informed consent was obtained from all patients.

All the patients received CSP, acupuncture and EA. After insertion of acupuncture needles, electrical stimulation was applied to initiate EA. All treatments were prepared and administered based on the standardized treatment protocol by a KM doctor with 20 years of clinical acupuncture experience. Using a sterile hypodermic syringe (30-gauge, 1.0 cc; DM Medicrat, Dongbang, Seoul, South Korea), $0.1 \mathrm{~mL}$ of CSP solution was administered into "acupoint PC7", which was around the transverse carpal ligament (TCL). In the supine position of hand, the needle was inserted perpendicularly to the skin toward PC7 at a depth of $0.5 \mathrm{~cm}$. A skin test was conducted prior to CSP treatment. CSP solutions were provided in a sterile laboratory at AJ Extramural Herbal Dispensary in accordance with Korea Good Manufacturing Practice (KGMP) under the supervision of a KM Doctor. Refining oil extracted from Cathami Semen (the seeds of Carthamus tinctorius L.) was used to manufacture the CSP solution [16]. After CSP injection, acupuncture was performed in all patients using sterile acupuncture (stainless steel, diameter $0.25 \mathrm{~mm} \times$ length $30 \mathrm{~mm}$, Dong Bang; Gyeonggi-do, South Korea). After the insertion of acupuncture, EA (CELLMAC, TSN-100; Gyeonggi-do, Korea) was performed at 2-Hz for $12 \mathrm{~min}$. The acupoints were PC7, PC6, SI5 and LI5 on the affected side.

For Outcome measurements, the visual analog scale (VAS) for pain and paresthesia (including numbness and tingling), Korean version of Boston carpal tunnel questionnaire (K-BCTQ) score [17], and changes in nocturnal pain, Tinel sign, and Phalen's test were measured before and after the treatments. Patients conducted satisfaction surveys at treatment completion.

Total 17 cases were included. The clinical characteristics of the study subjects at baseline are summarized in Table 1. Treat- 


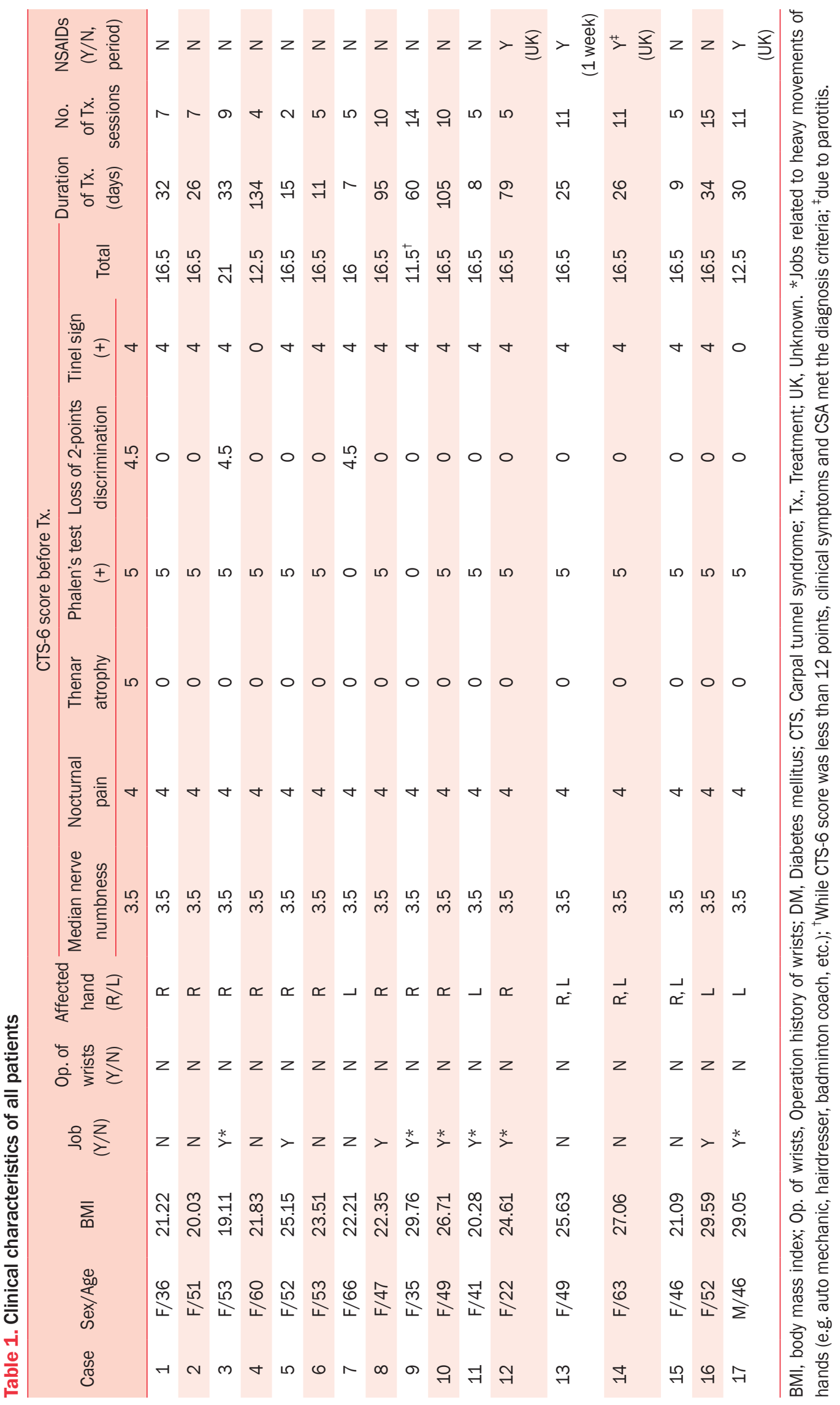




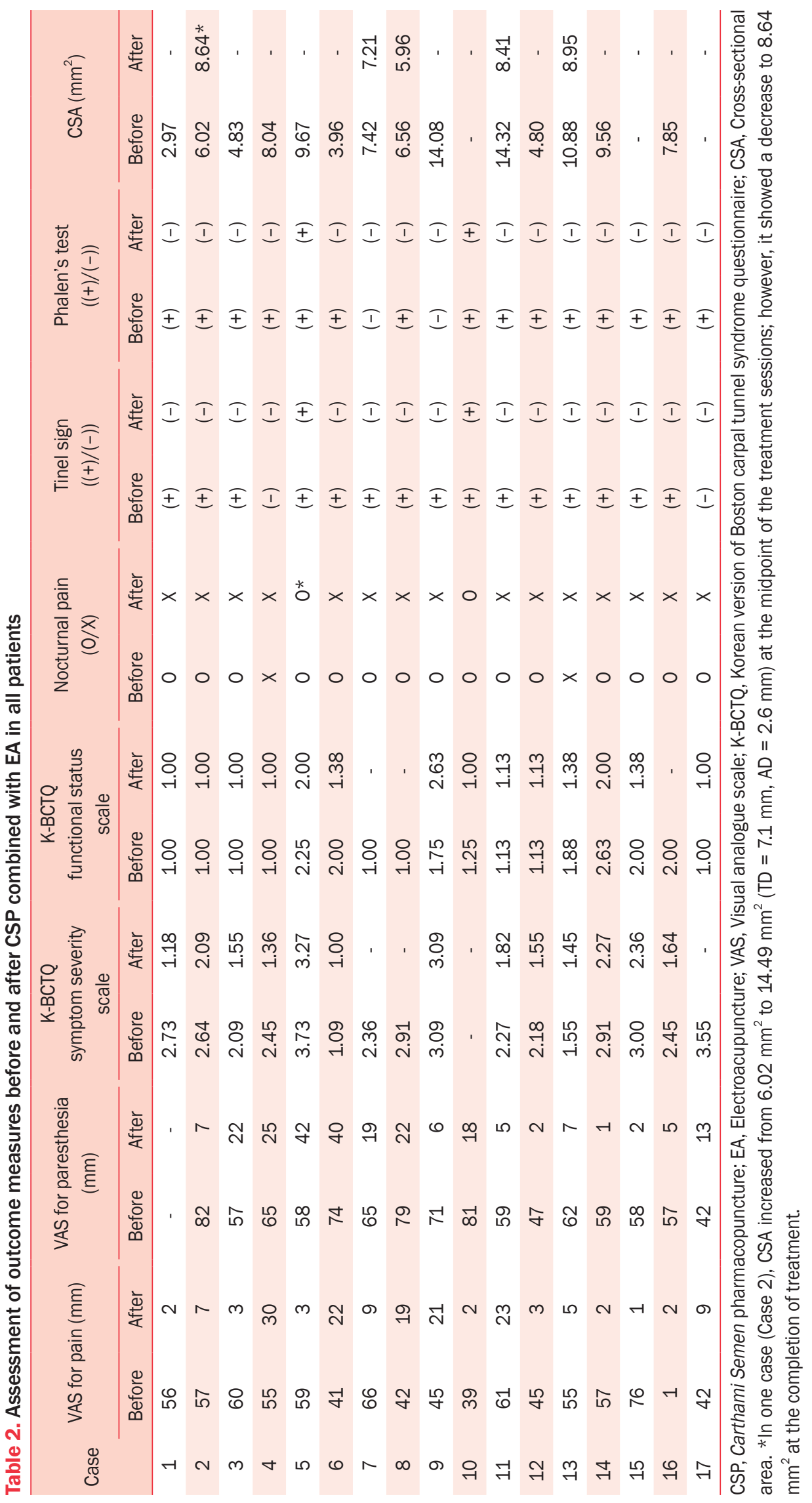


ment was terminated according to patients' improvement or KM doctor's judgement. The mean treatment duration was $42.88 \pm 38.14$ days and the mean number of treatment sessions was $8.00 \pm 3.71$. By the last treatment, the VAS for pain significantly decreased from $50.41 \pm 16.19$ to $9.59 \pm 9.46$, and VAS for paresthesia was also significantly reduced from $63.50 \pm 11.49$ to $14.75 \pm 12.97$ (all $\mathrm{p}<0.001$ ). K-BCTQ symptom severity scale declined from $2.48 \pm 0.68$ to $1.89 \pm 0.70$ ( $\mathrm{p}<0.001)$. However, no statistically significant change in functional status scores were observed after the treatment course. Nocturnal pain, Phalen's test and Tinel signs showed improvements after all the treatments (Table 2, 3).

Out of 17 patients, ultrasound observations were conducted in 14 patients before treatment for supporting diagnosis, and in only 5 patients after treatment. We observed changes in transverse diameter (TD), anteroposterior diameter (AD), and crosssectional area (CSA; $\left.\mathrm{TD}^{\star} \mathrm{AD}^{\star} \pi / 4\right)$ of the median nerve (Table 2 ). In 4 cases (Case $7,8,11,13$ ), CSA decreased upon completion of treatment. In 1 case (Case 2), CSA increased from $6.02 \mathrm{~mm}^{2}$ to $14.49 \mathrm{~mm}^{2}$ at the midpoint of the treatment sessions. How- ever, it showed a decrease to $8.64 \mathrm{~mm}^{2}$ after treatment. In Case 11 , a noticeable reduction in CSA was observed after treatment (Fig. 1).

No severe adverse events ( $\mathrm{AE}$ ) were detected during treatment. A few patients showed mild swelling and redness at acupoints, and subsided within 1-2 days.

The patient satisfaction survey was completed by 13 out of 17 participants (Table 4). All the respondents reported favorable overall satisfaction with KM treatments. Moreover, $72.7 \%$ of patients were open to future pharmacopuncture treatments if CTS recurred.

\section{DISCUSSION}

To the authors' knowledge, this is the first case series study that aimed to report the clinical effects of CSP combined with EA on CTS. In response to KM treatment, 17 patients showed significant improvements in pain, paresthesia, and all other related symptoms in the short-term period. Furthermore, the patients showed no AEs or complications.

Table 3. Statistical analysis* for outcome scores change before and after CSP combined with EA in all patients

\begin{tabular}{|c|c|c|c|c|}
\hline Variables & Before treatment & After treatment & Mean difference $(95 \% \mathrm{Cl})$ & $\mathrm{p}$-value \\
\hline VAS for pain $(n=17)$ & $50.41 \pm 16.19$ & $9.59 \pm 9.46$ & $40.82(31.18-50.47)$ & $<0.001$ \\
\hline VAS for paresthesia $(n=16)$ & $63.50 \pm 11.49$ & $14.75 \pm 12.97$ & $48.75(40.72-56.78)$ & $<0.001$ \\
\hline K-BCTQ symptom severity scale $(n=13)$ & $2.48 \pm 0.68$ & $1.89 \pm 0.70$ & $0.59(0.32-0.83)$ & $<0.001$ \\
\hline K-BCTQ functional status scale ${ }^{\dagger}(n=13)$ & $1.19[1.00,2.00]^{\ddagger}$ & $1.13[1.00,1.38]^{\ddagger}$ & $0.25[0,0.62]^{\ddagger}$ & 0.713 \\
\hline
\end{tabular}

CSP, Carthami Semen pharmacopuncture; VAS, visual analog scale; K-BCTQ, Korean version of Boston carpal tunnel syndrome questionnaire. *Data were analyzed using Statistical Package for Social Sciences (SPSS) version 22.0 for Windows (SPSS Inc., Chicago, IL, USA); ${ }^{\dagger}$ Wilcoxon signed-rank test was conducted due to non-normal distribution in score change; ${ }^{\ddagger}$ Median value [Quartile1, Quartile3].
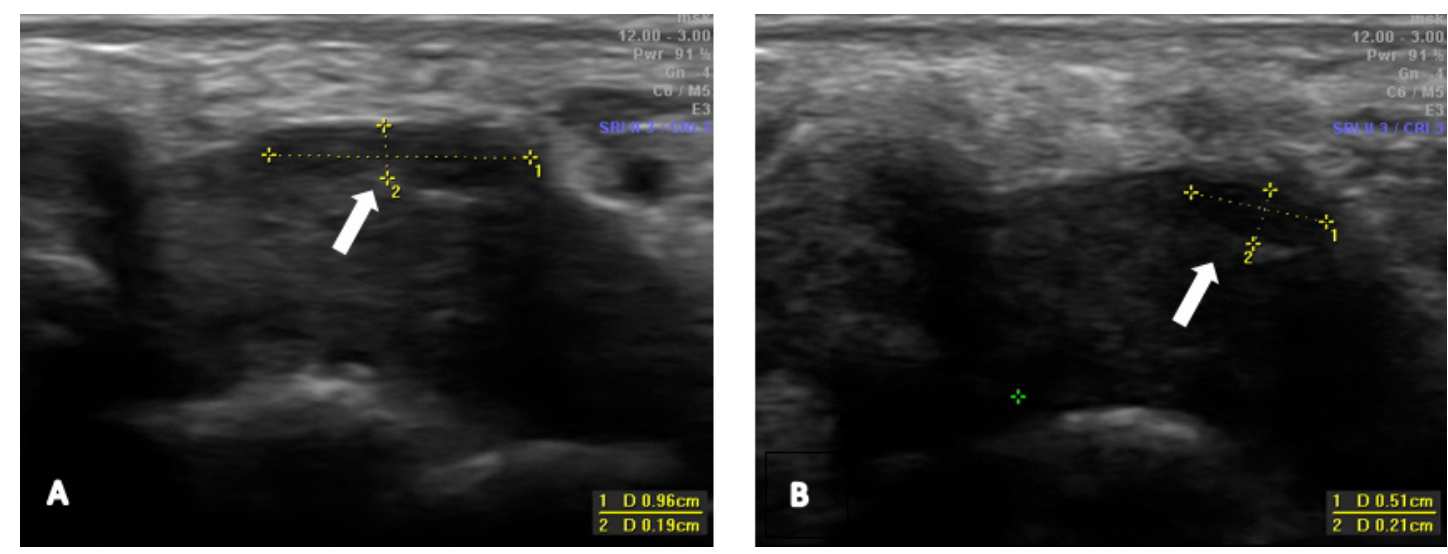

Figure 1. Transverse ultrasound images of the median nerve (arrows) in one patient (Case 11); (A) Before treatment, TD and AD were $9.6 \mathrm{~mm}$ and $1.9 \mathrm{~mm}$, respectively. $\mathrm{CSA}\left(=\mathrm{TD} * A D^{*} \pi / 4\right)$ was $14.32 \mathrm{~mm}^{2}$; (B) After treatment, the TD and AD decreased into $5.1 \mathrm{~mm}$ and $2.1 \mathrm{~mm}$, respectively. Accordingly, CSA was $8.41 \mathrm{~mm}^{2}$. 
Table 4. Patient satisfaction with KM treatment $(n=13)$

\begin{tabular}{|c|c|c|c|c|c|c|}
\hline Questions & $\begin{array}{c}\text { Strongly disagree } \\
1 \\
N(\%)\end{array}$ & $\begin{array}{c}\text { Disagree } \\
2 \\
N(\%)\end{array}$ & $\begin{array}{c}\text { Moderate } \\
3 \\
\mathrm{~N}(\%)\end{array}$ & $\begin{array}{c}\text { Agree } \\
4 \\
N(\%)\end{array}$ & $\begin{array}{c}\text { Strongly agree } \\
5 \\
N(\%)\end{array}$ & $\begin{array}{c}\text { Mean } \pm \text { SD } \\
(N=13)\end{array}$ \\
\hline $\begin{array}{l}\text { "I am satisfied with KM treatments } \\
\text { I have been given." }\end{array}$ & $0(0)$ & $0(0)$ & $9(69.23)$ & $4(30.77)$ & $0(0)$ & $3.31 \pm 0.48$ \\
\hline $\begin{array}{l}\text { "I have been given the } \\
\text { pharmacopuncture treatment in } \\
\text { a safe way." }\end{array}$ & $0(0)$ & $0(0)$ & $4(30.77)$ & $8(61.54)$ & 1 (7.69) & $3.77 \pm 0.60$ \\
\hline $\begin{array}{l}\text { "The pharmacopuncture treatment } \\
\text { improved my CTS symptoms." }\end{array}$ & $0(0)$ & $0(0)$ & $3(27.27)$ & $9(69.23)$ & 1 (7.69) & $3.85 \pm 0.55$ \\
\hline $\begin{array}{l}\text { "The other KM treatments (except } \\
\text { pharmacopunture) were helpful in } \\
\text { improving my CTS symptoms." }\end{array}$ & $0(0)$ & $0(0)$ & $2(18.18)$ & $11(23.08)$ & $0(0)$ & $3.85 \pm 0.38$ \\
\hline $\begin{array}{l}\text { "I will recommend pharmacopuncture } \\
\text { to others in the future." }\end{array}$ & $0(0)$ & $0(0)$ & $7(53.85)$ & $5(38.46)$ & 1 (7.69) & $3.54 \pm 0.66$ \\
\hline $\begin{array}{l}\text { "If CTS recurs, I will intend to be given } \\
\text { pharmacopuncture treatment." }\end{array}$ & $0(0)$ & $0(0)$ & $4(30.77)$ & $8(61.54)$ & $1(7.69)$ & $3.77 \pm 0.60$ \\
\hline
\end{tabular}

KM, Korean Medicine; CTS, Carpal tunnel syndrome.

KM treatments, such as acupuncture, pharmacopuncture, and electroacupuncture are effective as conservative treatments for musculoskeletal disorders like CTS in the absence of abnormalities like a tumor or post-fracture malunion [10]. Therefore, given the potential sequelae of steroid injections and surgical treatments, conservative treatments like KM can be recommended as initial treatment options. Phamacopuncture has been reported to have symptom-relieving effects in CTS [10-13]. CSP effects in CTS are likely due to a combination of pharmacological action and lubrication to the TCL [18]. Carthami Semen extract has been reported to induce analgesic and anti-inflammatory effects, and decrease the size of swelling at joints $[19,20]$. Although the mechanisms of CSP-mediatedalleviation of paresthesia and pain in CTS remain unknown, CSP may improve blood circulation around the TCL and digital flexor tendons, decrease wrist joint swelling, and ameliorate tendon inflammation.

For CTS patients, EA likely promotes pain inhibition and recovery stimulation. EA blocks pain by activating a variety of bioactive chemicals (i.e., opioids, serotonin, and norepinephrine) via peripheral, spinal, and supraspinal mechanisms. Opioids desensitizes peripheral nociceptors and reduces proinflammatory cytokines peripherally and in the spinal cord [21]. Moreover, serotonin and norepinephrine induced by EA decrease spinal N-methyl-d-aspartate receptor (NMDAR) subunit GluN1 phosphorylation, which modulates NMDAR activity and promotes transmission of nociceptive inputs [21, 22]. EA may also improve median nerve function at the wrist by somatotopically distinct neuroplasticity in the primary somatosensory cortex of the brain [23]. Despite these compelling results, a review from Cochrane [2] concluded that it indicated that EA-mediated pain reduction was short-term.

Typical CTS symptoms are comparable to Arthralgia syndrome (痺症, Bi Syndrome) in KM. There are three representative symptoms of Arthralgia syndrome, i.e., pain, numbness and dullness. Arthralgia syndrome is considered as a result of qi-blood circulation blockage and caused by pathogenic wind (風), cold dampness (寒濕), and blood stasis (瘀血) [13, 24]. In KM, Carthami Semen is considered to have warmness (溫 性) and promote blood circulation. Thus, in the concept of KM, CSP could help in influencing a blocked circulation of blood and removing the pathogenic wind, cold dampness, and blood stenosis, resulting in reduced pain and paresthesia related to Arthralgia syndrome [13].

In this study, besides mild redness and swelling, no severe AEs were detected. Furthermore, in another review, no severe AEs were associated with EA treatment for CTS [2]. Moreover, Choi et al. reported that water-soluble CSP did not induce any significant in vivo toxicological effects except localized symptoms [16]. However, it still needs more evidence to combine EA and CSP on CTS.

The present retrospective case series study has several limi- 
tations. First, this study is a case series with small sample size and a lack of control group; this indicates that it is limited to evaluate precisely the combinatorial effects of CSP and EA even though we can find the certain tendency of improvements in CTS symptoms after CSP combined with EA treatment. Hence, further prospective studies with large patients are needed. Second, all cases were limited to 'mild to moderate' CTS patients. Patients with surgical indications accompanied by thenar atrophy occasionally visit in KM Clinic, but there was no observation for the case in this study. Lastly, the study lacked followup assessments for evaluating a recurrence rate. CTS is prone to recurrence; thus, a follow-up assessment must be performed to evaluate the duration of treatment effects in future studies.

While this study has several limitations, we found that CSP combined with EA could be safe and helpful in improving CTS symptoms. Further prospective studies with larger populations, follow-up assessments, and intensive observations of adverse effects are needed.

\section{CONFLIGT OF INTEREST}

The authors declare that there are no conflicts of interest.

\section{FINANCIAL SUPPORT}

This study was supported by Korea Institute of Oriental Medicine (KSN2021210).

\section{ORCID}

Pyung-Wha Kim, https://orcid.org/0000-0002-8356-8392

Seon Choe, https://orcid.org/0000-0001-9504-2956

Kyungsun Han, https://orcid.org/0000-0002-9710-7845

Changsop Yang, https://orcid.org/0000-0003-1277-3329

Jinbok Lee, https://orcid.org/0000-0003-2725-4062

Sungha Kim, https://orcid.org/0000-0001-5542-3850

Minseop Shin, https://orcid.org/0000-0002-1698-8987

\section{REFERENCES}

1. Padua L, Coraci D, Erra C, Pazzaglia C, Paolasso I, Loreti C, et al. Carpal tunnel syndrome: clinical features, diagnosis, and management. Lancet Neurol. 2016;15(12):1273-84

2. Choi GH, Wieland LS, Lee H, Sim H, Lee MS, Shin BC. Acupuncture and related interventions for the treatment of symp- toms associated with carpal tunnel syndrome. Cochrane Database Syst Rev. 2018;12(12):CD011215.

3. Lee JW, Kim JY, Hong YS, Kim SS, Kim WC, Kim HJ. The prevalence of carpal tunnel syndrome in an urban population. J Korean Soc Surg Hand. 2008;13(4):266-75.

4. Sim H, Shin BC, Lee MS, Jung A, Lee H, Ernst E. Acupuncture for carpal tunnel syndrome: a systematic review of randomized controlled trials. J Pain. 2011;12(3):307-14.

5. Jerosch-Herold C, Houghton J, Blake J, Shaikh A, Wilson EC, Shepstone L. Association of psychological distress, quality of life and costs with carpal tunnel syndrome severity: a cross-sectional analysis of the PALMS cohort. BMJ Open. 2017;7(11):e017732.

6. American Academy of Orthopaedic Surgeons. Management of carpal tunnel syndrome evidence-based clinical practice guideline. Rosement: American Academy of Orthopaedic Surgeons; 2016. p. 7, 10-2, 407-8

7. Kaile E, Bland JDP. Safety of corticosteroid injection for carpal tunnel syndrome. J Hand Surg Eur Vol. 2018;43(3):296-302.

8. Bae HS, Hong JW, Choi CM, Na BJ, Park SU, Moon SK, et al. Comparison of therapeutic effect on carpal tunnel syndrome between oriental and western medicine. J Korean Orient Med. 2008;28(1):87-93.

9. Verdugo RJ, Salinas RA, Castillo JL, Cea JG. Surgical versus non-surgical treatment for carpal tunnel syndrome. Cochrane Database Syst Rev. 2008;2008(4):CD001552.

10. Ku JY, Lee KH, Cho SW, Lee SC, Youn HM, Jang KJ, et al. Comparison of the effects between sweet bee venom pharmacopuncture and scolopendrid pharmacopuncture on carpal tunnel syndrome (randomized, controlled clinical trial). J Pharmacopuncture. 2010;13(4):75-89.

11. Lee SH, Yoon JW, Kim SJ. A comparative review on carthmiflos pharmacopuncture treatment-focused on clinical study. J Korean Med Rehabil. 2017;27(4):55-65.

12. Choi SW, Park PB, Oh SJ. A case report of carpal tunnel syndrome with Raynaud's phenomenon treated by bee venom and carthami flos pharmacopuncture. J Pharmacopuncture. 2009;12(1):103-8.

13. Shin MS, Park MH, Lim ST, Shin JC, Lee BY, Lee SS, et al. Clinical study on treatment of the carpal tunnel syndrome with $\mathrm{CF}$ herbal acupuncture. J Pharmacopuncture. 2005;8(2):39-45.

14. Fowler JR, Cipolli W, Hanson T. A comparison of three diagnostic tests for carpal tunnel syndrome using latent class analysis. J Bone Joint Surg Am. 2015;97(23):1958-61.

15. Park GY. Ultrasound in the diagnosis of carpal tunnel syndrome. J Korean Assoc Pain Med. 2006;5(2):81-5.

16. Choi YM, Jung DJ, Kim SH, Kim JU, Yook TH. Repeated intramuscular-dose toxicity test of watersoluble carthami flos (WCF) pharmacopuncture in sprague-dawley rats. J Pharmacopunc- 
ture. 2015;18(1):36-43.

17. Park DJ, Kang JH, Lee JW, Lee KE, Wen L, Kim TJ, et al. Crosscultural adaptation of the Korean version of the Boston carpal tunnel questionnaire: its clinical evaluation in patients with carpal tunnel syndrome following local corticosteroid injection. J Korean Med Sci. 2013;28(7):1095-9.

18. Yook TH, Song BY, Sin MS, Kim BH, Park JJ, Yun JH. Effects on the local thermal change following herbal acupuncture on D.I.T.I. J Korean Acupunct Moxib Soc. 2000;17(3):57-68.

19. Seo BI, Lee ES, Park JH, Kim SC, Byeon BH, Choi HY. A efficacy of Korean and Chinese Carthami Semen on analgesic effect, anti-inflammation and arthritis. Korea J Herbol. 2001;16(1):110 .

20. Kim SH, Lee JM. Effects of aqua-acupuncture with carthami flos oil on gout in rats induced by microcrystalline sodium urate. J Korean Acupunct Moxib Soc. 1998;15(1):483-91.

21. Zhang R, Lao L, Ren K, Berman BM. Mechanisms of acupuncture-electroacupuncture on persistent pain. Anesthesiology. 2014;120(2):482-503

22. Zhang X, Wu J, Lei Y, Fang L, Willis WD. Protein phosphatase modulates the phosphorylation of spinal cord NMDA receptors in rats following intradermal injection of capsaicin. Brain Res Mol Brain Res. 2005;138(2):264-72.

23. Maeda Y, Kim H, Kettner N, Kim J, Cina S, Malatesta C, et al. Rewiring the primary somatosensory cortex in carpal tunnel syndrome with acupuncture. Brain. 2017;140(4):914-27.

24. Zhang EQ. Bi syndrome (arthralgia syndrome). J Tradit Chin Med. 2010;30(2):145-52. 